\title{
RUNNING MARKOV CHAIN WITHOUT MARKOV BASIS
}

\author{
H. HARA \\ Faculty of Economics, Niigata University, \\ Niigata, 950-2181, Japan \\ E-mail: hara@econ-niigata-u.ac.jp \\ S. AOKI \\ Department of Mathematics and Computer Science, Kagoshima University \\ Kagoshima, 890-0065, Japan \\ E-mail:aoki@sci.kagoshima-u.ac.jp \\ JST, CREST

\section{A. TAKEMURA} \\ Department of Mathematical Informatics, \\ Graduate School of Information Science and Technology, University of Tokyo, \\ Tokyo, 113-8656, Japan \\ E-mail: takemura@stat.t.u-tokyo.ac.jp \\ JST, CREST
}

The methodology of Markov basis initiated by Diaconis and Sturmfels ${ }^{1}$ stimulated active research on Markov bases for more than ten years. It also motivated improvements of algorithms for Gröbner basis computation for toric ideals, such as those implemented in 4 ti2. ${ }^{2}$ However at present explicit forms of Markov bases are known only for some relatively simple models, such as the decomposable models of contingency tables. Furthermore general algorithms for Markov bases computation often fail to produce Markov bases even for moderate-sized models in a practical amount of time. Hence so far we could not perform exact tests based on Markov basis methodology for many important practical problems.

In this article we propose to use lattice bases for performing exact tests, in the case where Markov bases are not known. Computation of lattice bases is much easier than that of Markov bases. With many examples we show that the approach with lattice bases is practical. We also check that its performance is comparable to Markov bases for the problems where Markov bases are known.

Keywords: exact test; lattice basis; MCMC. 


\section{Introduction}

Since Diaconis and Sturmfels ${ }^{1}$ introduced a Markov basis and proposed an algorithm of exact test by sampling contingency tables sharing a sufficient statistic, the algebraic and statistical properties of Markov bases for toric models have been extensively studied. Once a Markov basis is given, we can perform an exact test by using the basis. There exist algebraic algorithms to compute a Markov basis and a Markov basis of models for relatively small contingency tables can be computed by a computer algebra system such as $4 \mathrm{ti} 2 .^{2}$ However the computational cost of these algorithms is very high and at present it is difficult to compute a Markov basis for even moderate-sized models by such softwares in a practical amount of time.

For important models for applications we can investigate the structure of Markov bases for the model. In general, however, the structure is complicated and explicit forms Markov bases are known only for a few models such as the decomposable model, ${ }^{3}$ no-three-factor interaction model for relatively small tables. ${ }^{4}$ Considering the fact that an exact test is needed especially when the sample size is relatively small for the degrees of freedom of the model and the chi-square approximation of a test statistic is not accurate, these results at this point are not satisfactory from a practical viewpoint.

The set of contingency tables sharing a sufficient statistic is called a fiber. Markov basis is defined as a set of moves connecting every fiber. One reason for the complexity of Markov bases is that they guarantee the connectivity of every fiber. In practice, we only need to connect a fiber which a given data set belongs to. Sometimes we can find a useful subset of a Markov basis which has a simple structure and guarantees the connectivity of particular fibers. ${ }^{5-7}$ However, again, such a subset is not easy to obtain in general. ${ }^{8}$

In view of these difficulties with Markov bases, for performing exact tests we propose to use a lattice basis, which is a basis of the integer kernel of a configuration matrix, instead of a Markov basis. Computation of lattice bases is much easier than computation of Markov basis. With many examples we show that the proposed approach is practical. Note that a lattice basis itself does not guarantee the connectivity of every fiber. However every move is written as an integer combination of elements of a lattice basis. Hence, if we generate moves in such a way that every integer combination of elements of a lattice basis has a positive probability, then we can indeed guarantee the connectivity of every fiber.

When we run a Markov chain over a fiber, the transition probabilities can be easily adjusted by the standard Metropolis-Hastings procedure. 
Hence we can use any probability distribution for generating the moves, as long as every integer combination of elements of a lattice basis has a positive probability.

Based on the above observations, in this paper we discuss sampling of contingency tables by using a lattice basis. We propose simple algorithms for generating moves such that every move is generated with a positive probability by using a lattice basis. We can apply the proposed method to models whose Markov basis is not easy to compute and we show the usefulness of the proposed method through numerical experiments.

The organization of the this paper is as follows. In Section 2 we give a brief review on a Markov basis and lattice basis. In Section 3 we propose algorithms for generating moves by using lattice basis and in Section 4 we show the practicality and usefulness of the proposed method through numerical experiments.

\section{Markov basis and lattice basis}

In this section we give a brief review on a Markov basis and a lattice basis. Let $\boldsymbol{x}=\{x(\boldsymbol{i}), \boldsymbol{i} \in \mathcal{I}\}$ denote a contingency table, where $x(\boldsymbol{i})$ is a cell frequency for a cell $\boldsymbol{i}$ and $\mathcal{I}$ is the set of cells. When we order the elements of $\boldsymbol{x}$ appropriately, $\boldsymbol{x}$ is considered as an $|\mathcal{I}|$ dimensional column vector. Let $\boldsymbol{t}$ denote the vector of the sufficient statistic for a toric model. In a toric model there exists an integer matrix $A$ satisfying

$$
A \boldsymbol{x}=\boldsymbol{t} .
$$

$A$ is called a configuration matrix associated with the model. The set of contingency tables sharing $\boldsymbol{t}$ is called a fiber and denoted by $\mathcal{F}_{\boldsymbol{t}}$.

Consider a goodness-of-fit test for the model. When $\boldsymbol{t}$ is fixed, $\boldsymbol{x}$ is distributed exactly as a hypergeometric distribution over the fiber $\mathcal{F}_{\boldsymbol{t}}$. If we can enumerate the elements of the fiber, it is possible to evaluate a test statistic based on the exact hypergeometric distribution. In general the enumeration is infeasible and the evaluation of the distribution of a test statistic is done by sampling contingency tables.

Let

$$
\operatorname{ker}_{\mathbb{Z}} A=\operatorname{ker} A \cap \mathbb{Z}^{|\mathcal{I}|}=\left\{\boldsymbol{z} \in \mathbb{Z}^{|\mathcal{I}|} \mid A \boldsymbol{z}=0\right\}
$$

denote the integer kernel of $A$. An element of $\operatorname{ker}_{\mathbb{Z}} A$ is called a move for the model. By adding or subtracting a move $\boldsymbol{z}=\{z(\boldsymbol{i}), \boldsymbol{i} \in \mathcal{I}\} \in \operatorname{ker}_{\mathbb{Z}} A$, a contingency table $\boldsymbol{x}$ is transformed to a table in the same fiber $\boldsymbol{y}=\boldsymbol{x}+\boldsymbol{z}$, as long as $\boldsymbol{y}$ does not contain a negative cell. A finite set of moves $\mathcal{B}=\left\{\boldsymbol{z}_{1}, \ldots, \boldsymbol{z}_{M}\right\}$ 
is called a Markov basis if for every fiber all the states become mutually accessible by moves in $\mathcal{B}$. Consider an undirected graph $G_{\boldsymbol{t}, \mathcal{B}}$ whose vertices are the elements of a fiber $\mathcal{F}_{\boldsymbol{t}}$. We draw an edge between $\boldsymbol{x} \in \mathcal{F}_{\boldsymbol{t}}$ and $\boldsymbol{y} \in \mathcal{F}_{\boldsymbol{t}}$ if there exists $\boldsymbol{z} \in \mathcal{B}$ such that $\boldsymbol{y}=\boldsymbol{x}+\boldsymbol{z}$ or $\boldsymbol{y}=\boldsymbol{x}-\boldsymbol{z} . \mathcal{B}=\left\{\boldsymbol{z}_{1}, \ldots, \boldsymbol{z}_{M}\right\}$ is a Markov basis if and only if $G_{t, \mathcal{B}}$ is connected for all $\boldsymbol{t}$. In this way a Markov basis guarantees the connectivity of every fiber. Combined with the standard Metropolis-Hastings procedure, the connectivity enables us to sample contingency tables from an irreducible Markov chain whose stationary distribution is the hypergeometric distribution by Markov chain Monte Carlo (MCMC) method. Therefore once a Markov basis is obtained, we can evaluate the distribution of a test statistic of a conditional test based on the exact distribution.

A move $\boldsymbol{z}$ is written as a difference of its positive part and negative part as $\boldsymbol{z}=\boldsymbol{z}^{+}-\boldsymbol{z}^{-}$, where $z^{+}(\boldsymbol{i})=\max (z(\boldsymbol{i}), 0)$ and $z^{-}(\boldsymbol{i})=\max (-z(\boldsymbol{i}), 0)$, $\boldsymbol{i} \in \mathcal{I}$. Consider a binomial $\boldsymbol{p}^{\boldsymbol{z}^{+}}-\boldsymbol{p}^{\boldsymbol{z}^{-}}$corresponding to $\boldsymbol{z}$, where $\boldsymbol{p}^{\boldsymbol{z}^{ \pm}}=$ $\prod_{\boldsymbol{i} \in \mathcal{I}} p(\boldsymbol{i})^{z^{ \pm}(\boldsymbol{i})}$ and $p(\boldsymbol{i})$ are indeterminates. The degree of the binomial $\boldsymbol{p}^{\boldsymbol{z}^{+}}-\boldsymbol{p}^{\boldsymbol{z}^{-}}$is called the degree of $\boldsymbol{z}$. Let $I_{A}$ be the toric ideal associated with a configuration $A$. Then $\boldsymbol{p}^{\boldsymbol{z}^{+}}-\boldsymbol{p}^{\boldsymbol{z}^{-}} \in I_{A}$ if and only if $\boldsymbol{z}=\boldsymbol{z}^{+}-\boldsymbol{z}^{-}$ is a move. Algebraically a Markov basis is defined as a generator of the toric ideal $I_{A}$. A Gröbner basis of $I_{A}$ forms a Markov basis. ${ }^{1}$ A Markov basis or a Gröbner basis of models for relatively small contingency tables can be computed by a computer algebra system such as 4 ti2. ${ }^{2}$ However the computational cost is very high and for even moderate-sized models it is difficult to compute a Markov basis or Gröbner basis in a practical amount of time.

Let $d=\operatorname{dim} \operatorname{ker} A=|\mathcal{I}|-\operatorname{rank} A$ be the dimension of linear space spanned by the elements of $\operatorname{ker} A$ in $\mathbb{R}^{|\mathcal{I}|}$. It is a standard fact that the integer lattice $\operatorname{ker}_{\mathbb{Z}} A$ possesses a lattice basis $\mathcal{L}=\left\{\boldsymbol{z}_{1}, \ldots, \boldsymbol{z}_{d}\right\}$, such that every $\boldsymbol{z} \in \operatorname{ker}_{\mathbb{Z}} A$ is a unique integer combination of $\boldsymbol{z}_{1}, \ldots, \boldsymbol{z}_{d} \cdot{ }^{9}$ Given $A$, it is relatively easy to compute such a basis of $\operatorname{ker}_{\mathbb{Z}} A$ using the Hermite normal form of $A$.

Usually a lattice basis contains exactly $d$ elements. In this paper we allow redundancy of a lattice basis and call a finite set $\mathcal{L}$ of moves a lattice basis if every move is written by an integral combination of the elements of $\mathcal{L}$. As we mentioned it is relatively easy to compute a lattice basis for a given $A$. Also, for many statistical models, where a Markov basis is hard to obtain, we can more easily identify a lattice basis. An example of this is the Lawrence lifting discussed in Section 3.2.

Let $S$ be a polynomial ring and let $I_{\mathcal{L}}=\left\langle\boldsymbol{p}^{\boldsymbol{z}} \mid \boldsymbol{z} \in \mathcal{L}\right\rangle$ be the ideal 
generated by a lattice basis $\mathcal{L}$. The toric ideal $I_{A}$ is obtained from $I_{\mathcal{L}}$ by taking saturation ${ }^{10,11}$

$$
\begin{aligned}
I_{A} & =\left(I_{\mathcal{L}}:\left\langle\prod_{\boldsymbol{i} \in \mathcal{I}} x(\boldsymbol{i})\right\rangle\right) \\
& :=\left\{y \in S \mid\left(\prod_{\boldsymbol{i} \in \mathcal{I}} x(\boldsymbol{i})\right)^{m} y \in I_{\mathcal{L}} \text { for some } m>0\right\} .
\end{aligned}
$$

Intuitively this fact shows that when the frequency of each cell is sufficiently large, the fiber is connected by the lattice basis $\mathcal{L}$.

\section{Sampling contingency tables with a lattice basis}

In this section we propose algorithms to generate a move based on a lattice bases. We also give lattice bases for higher Lawrence configurations.

\subsection{Generating moves by using a lattice basis}

Assume that $\mathcal{L}=\left\{\boldsymbol{z}_{1}, \ldots, \boldsymbol{z}_{K}\right\}, K \geq d$, is a lattice basis. Then any move $\boldsymbol{z} \in \operatorname{ker}_{\mathbb{Z}} A$ is expressed as

$$
\boldsymbol{z}=\alpha_{1} \boldsymbol{z}_{1}+\cdots+\alpha_{K} \boldsymbol{z}_{K}, \quad \alpha_{1}, \ldots, \alpha_{K} \in \mathbb{Z} .
$$

Then we can generate a move $\boldsymbol{z}$ by generating the integer coefficients $\alpha_{1}, \ldots, \alpha_{K}$. In the numerical experiments in the next section we use the following two methods to generate $\alpha_{1}, \ldots, \alpha_{K}$. Both methods generate all integer combinations of elements of $\mathcal{L}$ with positive probabilities and hence guarantee the connectivity of all fibers.

\section{Algorithm 3.1.}

Step 1 Generate $\left|\alpha_{1}\right|, \ldots,\left|\alpha_{K}\right|$ from Poisson distribution with mean $\lambda$,

$$
\left|\alpha_{k}\right| \stackrel{\text { iid }}{\sim} \operatorname{Po}(\lambda)
$$

and exclude the case $\left|\alpha_{1}\right|=\cdots=\left|\alpha_{K}\right|=0$.

Step $2 \alpha_{k} \leftarrow\left|\alpha_{k}\right|$ or $\alpha_{k} \leftarrow-\left|\alpha_{k}\right|$ with probability $1 / 2$ for $k=1, \ldots, K$.

\section{Algorithm 3.2.}

Step 1 Generate $|\alpha|=\sum_{i=1}^{K}\left|\alpha_{i}\right|$ from geometric distribution with parameter $p$

$$
|\alpha| \sim \operatorname{Geom}(p)
$$




$$
\begin{gathered}
\text { and allocate }|\alpha| \text { to } \alpha_{1}, \ldots, \alpha_{K} \text { according to multinomial distribution } \\
\alpha_{1}, \ldots, \alpha_{K} \sim \operatorname{Mn}(|\alpha| ; 1 / K, \ldots, 1 / K)
\end{gathered}
$$

Step $2 \alpha_{k} \leftarrow\left|\alpha_{k}\right|$ or $\alpha_{k} \leftarrow-\left|\alpha_{k}\right|$ with probability $1 / 2$ for $k=1, \ldots, K$.

\subsection{A lattice basis for higher Lawrence configuration}

Consider a configuration matrix of the form

$$
\Lambda(A)=\left(\begin{array}{ll}
A & 0 \\
I & I
\end{array}\right)
$$

where $I$ is an identity matrix. $\Lambda(A)$ is called the Lawrence lifting of $A$ or a Lawrence configuration. ${ }^{12}$ More generally the $r$-th Lawrence configuration is defined by

$$
\Lambda^{(r)}(A)=\left(\begin{array}{ccccc}
\overbrace{A} & 0 & \cdots & 0 & 0 \\
0 & A & 0 & \cdots & 0 \\
\vdots & \ddots & \ddots & \ddots & \vdots \\
0 & \cdots & 0 & A & 0 \\
I & I & \cdots & I & I
\end{array}\right) .
$$

Many practical statistical models including the no-three-factor interaction model and the discrete logistic regression model discussed in the following section have Lawrence configurations. In general a Markov basis for the Lawrence configuration is very difficult to compute. ${ }^{6,7}$ On the other hand it is easy to compute a lattice basis and the proposed method is available even for such models. We can compute a lattice basis of $\Lambda^{(r)}(A)$ by the following propositions.

Proposition 3.1. Let the column vectors of a matrix $B$ form a lattice basis of $A$. Then the column vectors of $\left(\begin{array}{c}B \\ -B\end{array}\right)$ form a lattice basis of $\Lambda(A)$.

Proof. Let $\boldsymbol{x}$ and $\boldsymbol{y}$ be two contingency tables in the same fiber for $\Lambda(A)$. Let $|\mathcal{I}|=2 n$ be the number of cells. Then we note that $n$ is the number of columns of $A$. Write $\boldsymbol{x}=\left(\boldsymbol{x}_{1}^{\prime}, \boldsymbol{x}_{2}^{\prime}\right)^{\prime}$, where $\boldsymbol{x}_{1}$ and $\boldsymbol{x}_{2}$ are $n \times 1$ column vectors and ' denotes the transpose. In the same way, write $\boldsymbol{y}=\left(\boldsymbol{y}_{1}^{\prime}, \boldsymbol{y}_{2}^{\prime}\right)^{\prime}$. Let

$$
z=\left(\begin{array}{l}
z_{1} \\
z_{2}
\end{array}\right)=x-y=\left(\begin{array}{l}
x_{1}-y_{1} \\
x_{2}-y_{2}
\end{array}\right)
$$


be a move of $\Lambda(A)$. Since $A \boldsymbol{z}_{1}=0, \boldsymbol{z}_{1}$ is written by an integer linear combination of $B$ as $\boldsymbol{z}_{1}=B \boldsymbol{\alpha}$, where $\boldsymbol{\alpha}$ is an $l \times 1$ integer vector. $\boldsymbol{z}_{0}+\boldsymbol{z}_{1}=0$ implies that $\boldsymbol{z}_{1}=-B \boldsymbol{\alpha}$ and therefore

$$
\boldsymbol{z}=\left(\begin{array}{c}
\boldsymbol{z}_{1} \\
\boldsymbol{z}_{2}
\end{array}\right)=\left(\begin{array}{c}
B \\
-B
\end{array}\right) \boldsymbol{\alpha}
$$

Hence $\left(\begin{array}{c}B \\ -B\end{array}\right)$ form a lattice basis of $\Lambda(A)$.

Proposition 3.2. Let the column vectors of $B$ form a lattice basis of $A$. Then the column vectors of

$$
B^{(r)}=\left(\begin{array}{cccc}
\overbrace{B} & 0 & \cdots & 0 \\
0 & B & \ddots & \vdots \\
\vdots & \ddots & \ddots & 0 \\
0 & \cdots & 0 & B \\
-B & -B & \cdots & -B
\end{array}\right) .
$$

form a lattice basis of higher Lawrence configuration $\Lambda^{(r)}(A)$.

Proof. We can interpret the $r$-th Lawrence lifting as $r$ slices of the original contingency table corresponding to $A$. The number of the cells for $\Lambda^{(r)}(A)$ is $|\mathcal{I}|=r n$, where $n$ is the number of cells (columns) of $A$. Let

$$
\boldsymbol{z}=\left(\begin{array}{c}
\boldsymbol{z}_{1} \\
\vdots \\
\boldsymbol{z}_{r}
\end{array}\right)=\boldsymbol{x}-\boldsymbol{y}=\left(\begin{array}{c}
\boldsymbol{x}_{1}-\boldsymbol{y}_{1} \\
\vdots \\
\boldsymbol{x}_{r}-\boldsymbol{y}_{r}
\end{array}\right)
$$

be a move of $\Lambda^{(r)}(A)$. We can express $\boldsymbol{z}_{1}=B \boldsymbol{\alpha}_{1}$. Then using the $r$-th slice as "pivots" we can write

$$
\boldsymbol{z}=\left(\begin{array}{c}
B \\
0 \\
\vdots \\
0 \\
-B
\end{array}\right) \boldsymbol{\alpha}_{1}+\left(\begin{array}{c}
0 \\
\boldsymbol{z}_{2} \\
\vdots \\
\boldsymbol{z}_{r-1} \\
\boldsymbol{z}_{r}+B \boldsymbol{\alpha}_{1}
\end{array}\right)
$$

Note that the first block of $\boldsymbol{z}$ is now eliminated. Performing the same operation recursively to other blocks we are left with the $(r-1)$-th slice and $r$-th slice, which is the same as the previous proposition. 
In this proposition we only used the last slice as pivots. More symmetric lattice basis can be obtained by columns of all pairwise differences of slices, for example for $r=3$

$$
\left(\begin{array}{ccc}
B & B & 0 \\
-B & 0 & B \\
0 & -B & -B
\end{array}\right)
$$

The lattice bases in the above propositions may contain redundant elements. However the set of moves including redundant elements are sometimes preferable for moving around the fiber. In general the computation of a lattice basis of $A$ is easier than the computation of a lattice basis of $\Lambda^{(r)}(A)$. Sometimes we can compute a Markov basis for $A$ even when it is difficult to compute a Markov basis of $\Lambda^{(r)}(A)$. If a Markov basis for $A$ is known, we can use it as a lattice basis for $A$ and apply the above propositions for obtaining a lattice basis of $\Lambda^{(r)}(A)$. In the following numerical experiments we compute a lattice basis by using the above propositions.

\section{Numerical experiments}

In this section we apply the proposed method to the no-three-factor interaction model and the discrete logistic regression model and show the usefulness of the proposed method.

\subsection{No-three-factor interaction model}

No-three-factor interaction model is a model for three-way contingency tables. Let $x_{i_{1} i_{2} i_{3}}$ and $p_{i_{1} i_{2} i_{3}}$ denote a cell frequency and a cell probability of a cell $\boldsymbol{i}=\left(i_{1}, i_{2}, i_{3}\right)$ of a three-way contingency table, respectively. Then the model is described as

$$
\log p_{i_{1} i_{2} i_{3}}=\mu_{12}\left(i_{1} i_{2}\right)+\mu_{23}\left(i_{2} i_{3}\right)+\mu_{31}\left(i_{3} i_{1}\right)
$$

where $\mu_{12}, \mu_{23}$ and $\mu_{31}$ are free parameters. Aoki and Takemura ${ }^{4}$ discussed the structure of Markov basis for $3 \times 3 \times K$ table in detail and showed that there exists a Markov basis such that the largest degree of moves is 10 . In general, however, the structure of Markov bases for this model is known to be complicated and the closed form expression of Markov bases for this model of general tables is not yet obtained at present. Even by using 4ti2, it is difficult to compute a Markov basis for contingency tables larger than $5 \times 5 \times 5$ tables within a practical amount of time. 
This model has the higher Lawrence configuration in (1) such that $A$ is a configuration for the two-way complete independence model. The set of basic moves of form

\begin{tabular}{|c|c|c|}
\hline & $i_{1}$ & $i_{1}^{\prime}$ \\
\hline$i_{2}$ & 1 & -1 \\
\hline$i_{2}^{\prime}$ & -1 & 1 \\
\hline
\end{tabular}

is known to be a Markov basis for the two-way complete independence model. By using this fact and Proposition 3.2, we can compute a lattice basis as a set of degree four moves,

\begin{tabular}{|c|c|c|}
\hline & $i_{1}$ & $i_{1}^{\prime}$ \\
\hline$i_{2}$ & 1 & -1 \\
\hline$i_{2}^{\prime}$ & -1 & 1 \\
\hline
\end{tabular} \mid \begin{tabular}{|c|c|c|}
\hline & $i_{1}$ & $i_{1}^{\prime}$ \\
\hline$i_{2}$ & -1 & 1 \\
\hline$i_{2}^{\prime}$ & 1 & -1 \\
\hline
\end{tabular}

In this experiment we compute an exact distribution of the log-likelihood ratio (LR) statistic of the goodness-of-fit test for no-three-factor interaction model against the three-way saturated model

$$
\log p_{i_{1} i_{2} i_{3}}=\mu_{123}\left(i_{1} i_{2} i_{3}\right) .
$$

We computed sampling distribution of the LR statistic for $I \times I \times I, I=$ $3,5,10$ three-way contingency tables. Then the degrees of freedom of the asymptotic $\chi^{2}$ distribution of LR statistic is $(I-1)^{3}$. We set the sample size as $5 I^{3}$. For $3 \times 3 \times 3$ tables, the number of burn-in samples and iterations are (burn-in, iteration $)=(1000,10000)$. In $3 \times 3 \times 3$ tables, a minimal Markov basis is known ${ }^{4}$ and we also compute a sampling distribution by a Markov basis. In other cases, we set (burn-in, iteration) $=(10000,100000)$.

Figure 1 presents the results for $3 \times 3 \times 3$ tables. Left, center and right figures are histograms, paths and correlograms of the LR statistic, respectively. Solid lines in the left figures are asymptotic $\chi^{2}$ distributions with degrees of freedom 8. $\alpha_{k}$ is generated from $\operatorname{Po}(\lambda), \lambda=1,10,50$.

We can see from the figures that the proposed methods show comparative performance to the sampling with a Markov basis. Although the sampling distribution and the path is somewhat unstable for $\lambda=50$, in other cases the sampling distributions are similar and the paths are stable after burn-in period. Unless we set $\lambda$ as extremely high, the proposed method is robust against the distribution of $\alpha_{k}$.

Figure 2 presents the results for $5 \times 5 \times 5$ and $10 \times 10 \times 10$ tables. In these cases Markov basis cannot be computed via 4 ti2 within a practical amount of time by an Intel Core 2 Duo $3.0 \mathrm{GHz}$ CPU machine. So we 
compute sampling distributions by the proposed method. For $5 \times 5 \times 5$ tables, $\alpha_{1}, \ldots, \alpha_{K}$ are generated from $\operatorname{Geom}(p), p=0.1,0.5$. The degrees of freedom of the asymptotic $\chi^{2}$ distribution is 64 . Also in this case we can see that the proposed methods perform well. The approximation of the sampling distributions to the asymptotic $\chi^{2}$ distribution is good and the paths are stable after burn-in period.

For $10 \times 10 \times 10$ tables, $\alpha_{1}, \ldots, \alpha_{K}$ are generated from $\operatorname{Po}(\lambda), \lambda=10,50$. The degrees of freedom of the asymptotic $\chi^{2}$ distribution is 729 . In this case the performances of the proposed methods look less stable. We also compute the cases where the sample sizes are $10 I^{3}$ and $100 I^{3}$ but the results are similar. This is considered to be because the size of fibers of $10 \times 10 \times 10$ tables is far larger than those of $3 \times 3 \times 3$ or $5 \times 5 \times 5$ tables and it is more difficult to move around all over a fiber. Even if we use a Markov basis, the result might not be improved. Increasing the number of iterations might lead to a better performance.

Comparing the paths with $\lambda=10$, the path with $\lambda=50$ looks relatively more stable. For larger tables, larger $\lambda$ might be preferable to move around a fiber.

\subsection{Discrete logistic regression model}

The logistic regression model with discrete covariates is considered as a model for contingency tables. The model is defined by the conditional probability for the response variable. The model with one covariate and the model with two covariates are described as

$$
p_{i_{1} \mid i_{2}}= \begin{cases}\frac{\exp \left(\mu_{i_{1}}+\alpha_{i_{1}} i_{2}\right)}{1+\sum_{i_{1}^{\prime}=1}^{I_{1}-1} \exp \left(\mu_{i_{1}^{\prime}}+\alpha_{i_{1}^{\prime}} i_{2}\right)}, & i_{1}=1, \ldots, I_{1}-1, \\ \frac{1}{1+\sum_{i_{1}^{\prime}=1}^{I_{1}-1} \exp \left(\mu+\alpha_{i_{1}^{\prime}} i_{2}\right)}, & i_{1}=I_{1},\end{cases}
$$

where $i_{2} \in \mathcal{I}_{2}$ and

$$
p_{i_{1} \mid i_{2} i_{3}}= \begin{cases}\frac{\exp \left(\mu_{i_{1}}+\alpha_{i_{1}} i_{2}+\beta_{i_{1}} i_{3}\right)}{1+\sum_{i_{1}^{\prime}=1}^{I_{1}-1} \exp \left(\mu_{i_{1}^{\prime}}+\alpha_{i_{1}^{\prime}} i_{2}+\beta_{i_{1}^{\prime}} i_{3}\right)}, & i_{1}=1, \ldots, I_{1}-1, \\ \frac{1}{1+\sum_{i_{1}^{\prime}=1}^{I_{1}-1} \exp \left(\mu_{i_{1}^{\prime}}+\alpha_{i_{1}^{\prime}} i_{2}+\beta_{i_{1}^{\prime}} i_{3}\right)}, & i_{1}=I_{1},\end{cases}
$$

where $\left(i_{2}, i_{3}\right) \in \mathcal{I}_{2} \times \mathcal{I}_{3}$, respectively. $p_{i_{1} \mid i_{2}}$ and $p_{i_{1} \mid i_{2} i_{3}}$ are conditional probabilities that the value of the response variable equals $i_{1}$ given the covariates $i_{2}$ and $\left(i_{2}, i_{3}\right)$, respectively. $\mathcal{I}_{2}$ and $\mathcal{I}_{2} \times \mathcal{I}_{3}$ are designs for covariates. The structure of Markov bases for discrete logistic regression model is also 
known to be complicated even for the case of binary responses $I_{1}=2{ }^{6,7}$ Chen et al. ${ }^{6}$ and Hara et al. ${ }^{7}$ discussed the model with one covariate which is discrete and equally spaced and showed that the set of degree four moves of form

\begin{tabular}{|c|c|c|c|}
\hline & 1 & -1 & $\begin{array}{ll}-1 & 1\end{array}$ \\
\hline & -1 & 1 & $1-1$ \\
\hline
\end{tabular}

connects all fibers. Hara et $\mathrm{al}^{7}$ generalized the argument to the model with two covariates both of which are equally spaced. However it seems to be difficult to generalize these arguments to the models with more than two covariates or with more than two responses $I_{1}>2$ at this point. A Markov basis connecting all designs has to contain higher degree moves and the number of moves in a Markov basis is very large. Table 1 presents the highest degrees and the numbers of moves in the minimal Markov bases of binomial logistic regression models with one covariate computed by 4 ti2. Even for models with one covariate, if a covariate has more than 20 levels, it is difficult to compute Markov bases of models via 4ti2 within a practical amount of time by a computer with a 32-bit processor.

The logistic regression model with $r$ responses has the $r$-th Lawrence configuration (1) where $A$ is a configuration for Poisson regression model. The computation of Markov bases of Poisson regression model is relatively easy. Therefore a lattice basis can be computed by Proposition 3.2 and we can apply the proposed method to these models.

Table 1. The highest degrees and the number of moves in a minimal Markov basis for binomial logistic regression models with one covariate

\begin{tabular}{lccccccc}
\hline & \multicolumn{8}{c}{ number of levels of a covariate } \\
& 10 & 11 & 12 & 13 & 14 & 15 & 16 \\
\hline maximum degree & 18 & 20 & 22 & 24 & 26 & 28 & 30 \\
number of moves & 1830 & 3916 & 8569 & 16968 & 34355 & 66066 & 123330 \\
\hline
\end{tabular}

In the experiment we considered the goodness-of-fit test of binomial or trinomial logistic regression model with two covariates against a model with three covariates

$p_{i_{1} \mid i_{2} i_{3} i_{4}}= \begin{cases}\frac{\exp \left(\mu_{i_{1}}+\alpha_{i_{1}} i_{2}+\beta_{i_{1}} i_{3}\right)+\gamma_{i_{1}} i_{4}}{1+\sum_{i_{1}^{\prime}=1}^{I_{1}-1} \exp \left(\mu_{i_{1}^{\prime}}+\alpha_{i_{1}^{\prime}} i_{2}+\beta_{i_{1}^{\prime}} i_{3}+\gamma_{i_{1}^{\prime}} i_{4}\right)}, & i_{1}=1, \ldots, I_{1}-1, \\ \frac{1}{1+\sum_{i_{1}^{\prime}=1}^{I_{1}-1} \exp \left(\mu_{i_{1}^{\prime}}+\alpha_{i_{1}^{\prime}} i_{2}+\beta_{i_{1}^{\prime}} i_{3}+\gamma_{i_{1}^{\prime}} i_{4}\right)}, & i_{1}=I_{1},\end{cases}$ 
where $\left(i_{2}, i_{3}\right) \in \mathcal{I}_{2} \times \mathcal{I}_{3}, i_{4} \in \mathcal{I}_{4}$. We use the LR statistic as a test statistic. We assume that $\mathcal{I}_{2} \times \mathcal{I}_{3}$ are $4 \times 4$ and $10 \times 10$ checkered designs as described in the following figure for the $4 \times 4$ case, where only $\left(i_{2}, i_{3}\right)$ in dotted patterns have positive frequencies.

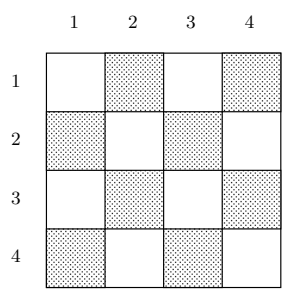

We also assume that $\mathcal{I}_{4}=\{1,2,3,4,5\}$. The degrees of freedom of the asymptotic $\chi^{2}$ distribution of the LR statistic is 1 . We set the sample sizes for $4 \times 4$ and $10 \times 10$ designs are 200 and 625 , respectively. We also set (burn-in, iteration $)=(1000,10000)$.

Figures 3 and 4 present the results for a binomial and a trinomial logistic regression models with $4 \times 4$ checkered pattern, respectively. Solid lines in the left figures are asymptotic $\chi^{2}$ distributions. $\alpha_{k}$ is generated from $\operatorname{Po}(\lambda)$, $\lambda=1,10,50$. We can compute Markov bases in these models. So we also present the results for Markov bases. We can see from the figures that the proposed methods show comparative performance to a Markov basis also in these models. We note that the paths are also stable even for the case where $\alpha_{1}, \ldots, \alpha_{K}$ are generated from $\operatorname{Po}(50)$.

Figure 5 presents the results for $10 \times 10$ checkered pattern. In this case Markov bases cannot be computed via 4 ti2 2 by our machine. $\alpha_{k}$ is generated from $\operatorname{Geom}(p), \lambda=0.1,0.5$. Also in these cases the results look stable. These results shows that the proposed method is useful for the logistic regression models for which that it is difficult to compute a Markov basis.

\section{References}

1. P. Diaconis and B. Sturmfels, Ann. Statist. 26, 363 (1998).

2. 4ti2 team, 4ti2 - a software package for algebraic, geometric and combinatorial problems on linear spaces Available at www.4ti2.de.

3. A. Dobra, Bernoulli 9, 1093 (2003).

4. S. Aoki and A. Takemura, Aust. N. Z. J. Stat. 45, 229 (2003).

5. S. Aoki and A. Takemura, Journal of Statistical Computation and Simulation 75, 787 (2005).

6. Y. Chen, I. Dinwoodie, A. Dobra and M. Huber, Lattice points, contingency tables, and sampling, in Integer points in polyhedra-geometry, number the- 
ory, algebra, optimization, , Contemp. Math. Vol. 374 (Amer. Math. Soc., Providence, RI, 2005) pp. 65-78.

7. H. Hara, A. Takemura and R. Yoshida, J. Multivariate Anal. 101, 909 (2010).

8. Y. Chen, I. Dinwoodie and R. Yoshida, Markov chain, quotient ideals and connectivity with positive margins, in Algebraic and Geometric Methods in Statistics, eds. P. Gibilisco, E. Riccomagno, M. P. Rogantin and H. P. Wynn (Cambridge Univ. Press, Cambridge, 2008) pp. 99-110.

9. A. Schrijver, Theory of linear and integer programming (John Wiley \& Sons Ltd., Chichester, 1986)

10. B. Sturmfels, Gröbner Bases and Convex Polytopes, University Lecture Series, Vol. 8 (American Mathematical Society, Providence, RI, 1996).

11. E. Miller and B. Sturmfels, Combinatorial Commutative Algebra, Graduate Texts in Mathematics, Vol. 227 (Springer-Verlag, New York, 2005).

12. F. Santos and B. Sturmfels, J. Combin. Theory Ser. A 103, 151 (2003). 

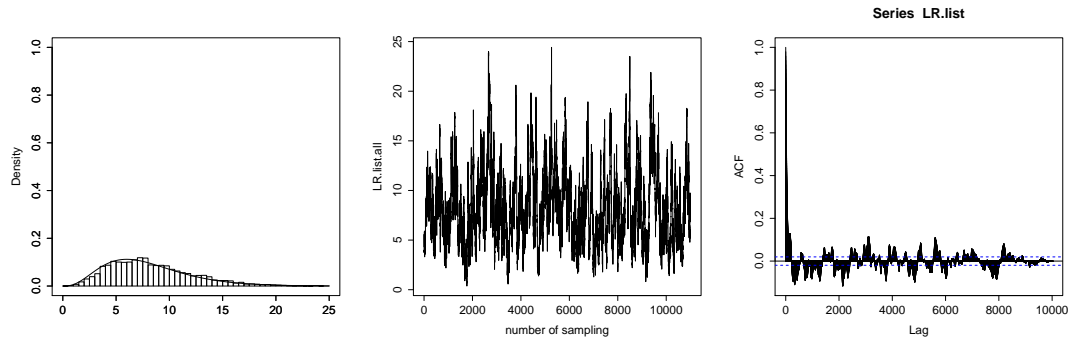

(a) a Markov basis
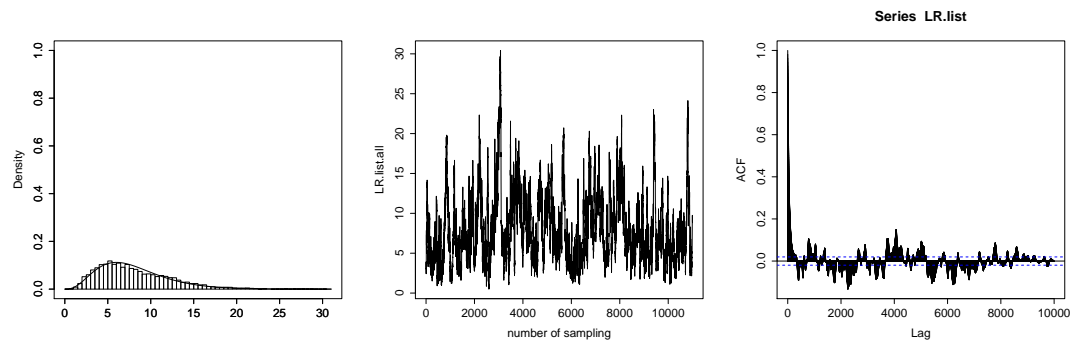

(b) a lattice basis with $P o(1)$
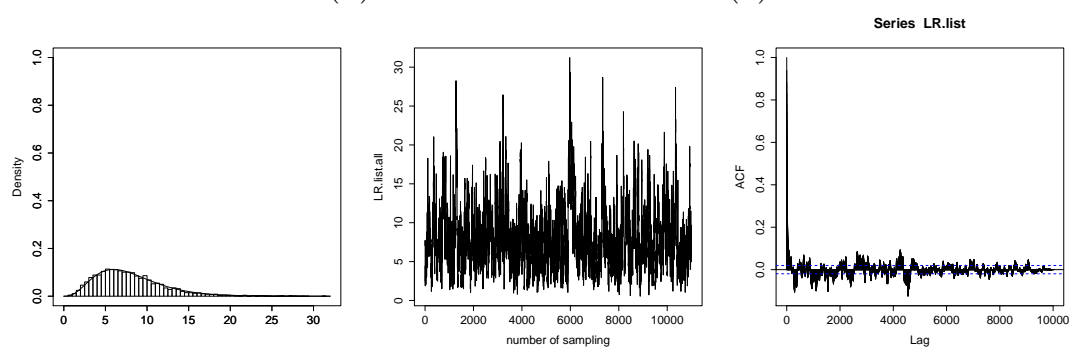

(c) a lattice basis with $P o(10)$
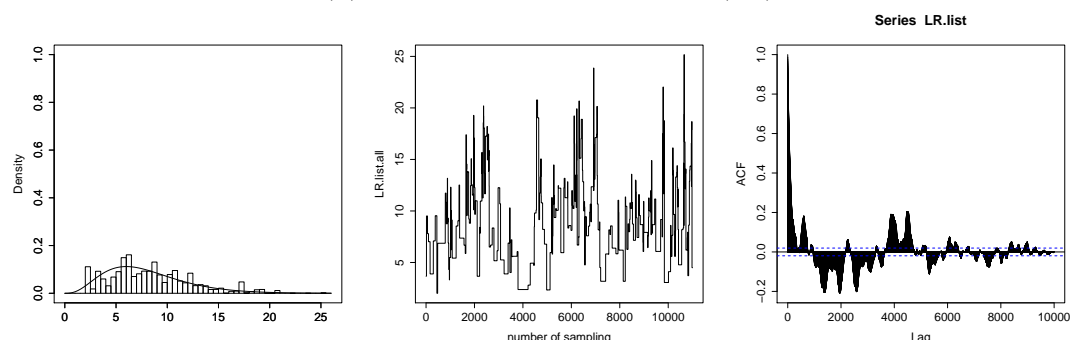

(d) a lattice basis with $P o(50)$

Fig. 1. Histograms, paths of LR statistic and correlograms for $3 \times 3 \times 3$ no-three-factor interaction model $(($ burn in,iteration $)=(1000,10000))$ 

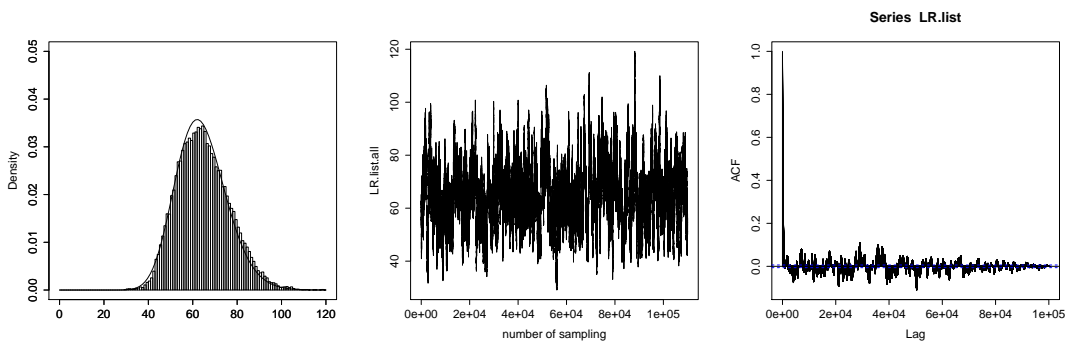

(a) $5 \times 5 \times 5$, a lattice basis with $\operatorname{Geom}(0.1)$
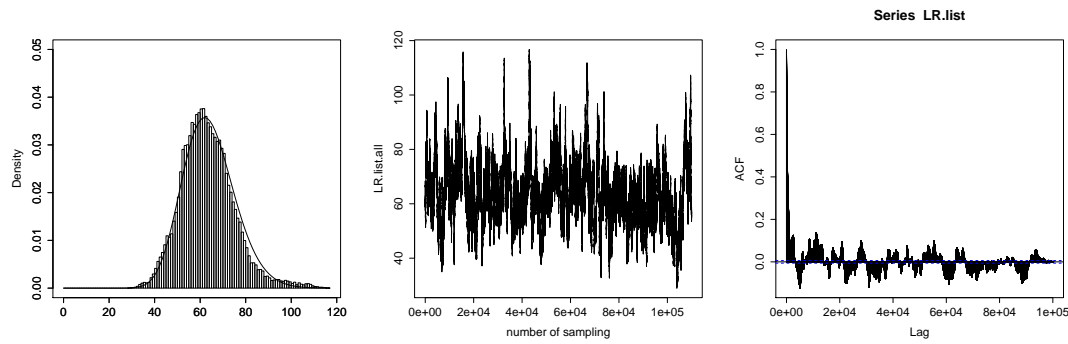

(b) $5 \times 5 \times 5$, a lattice basis with $\operatorname{Geom}(0.5)$
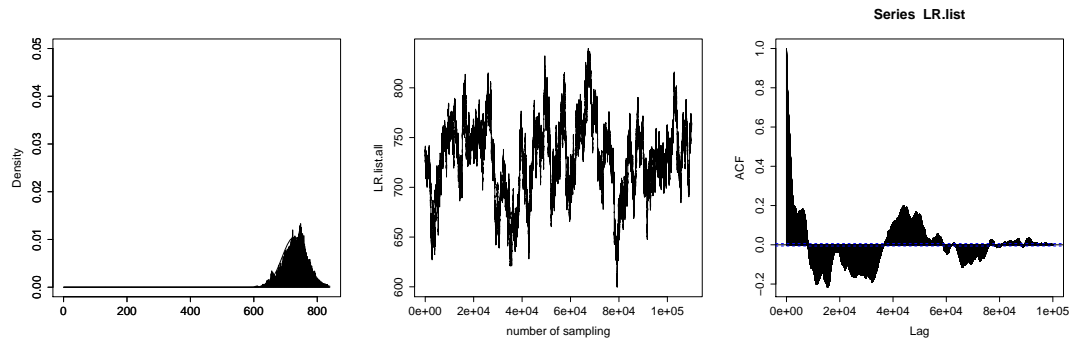

(c) $10 \times 10 \times 10$, a lattice basis with $P o(10)$
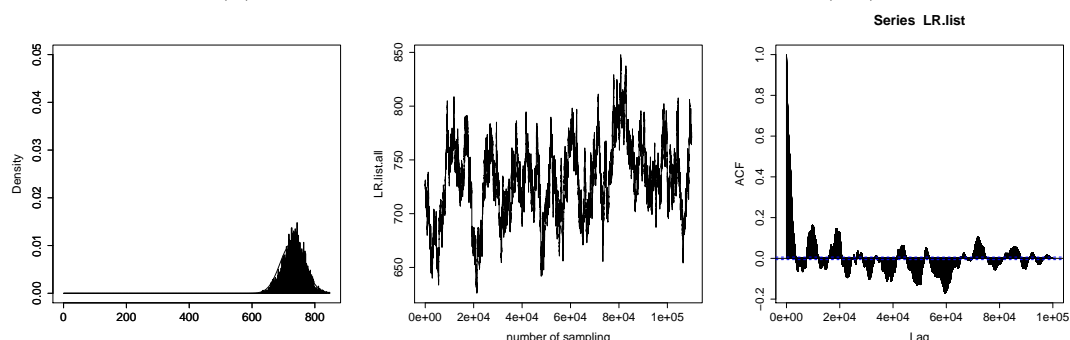

(d) $10 \times 10 \times 10$, a lattice basis with $P o(50)$

Fig. 2. Histograms, paths of LR statistic and correlograms of paths for no-three-factor interaction model $(($ burn in,iteration $)=(10000,100000))$ 

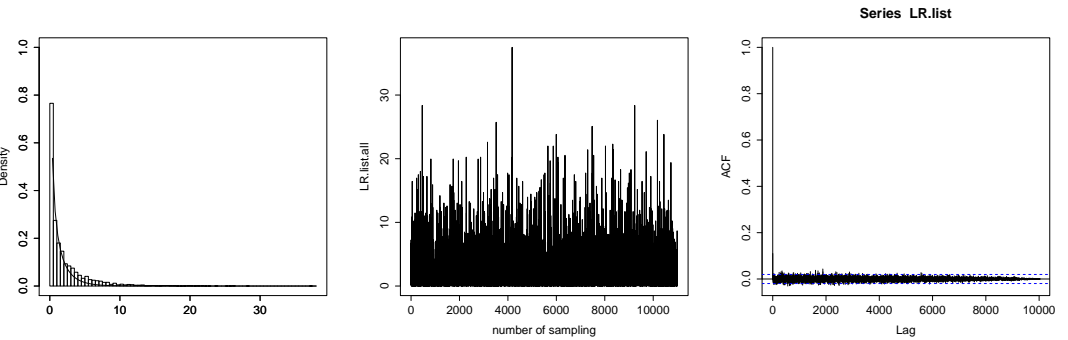

(a) a Markov basis
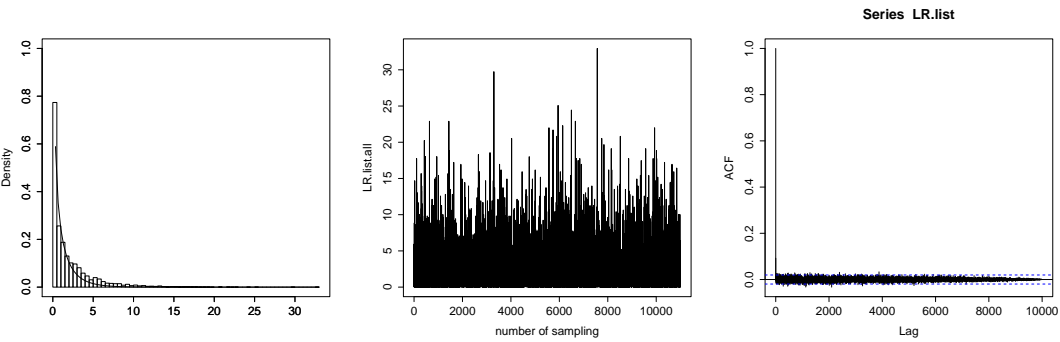

(b) a lattice basis with $P o(1)$
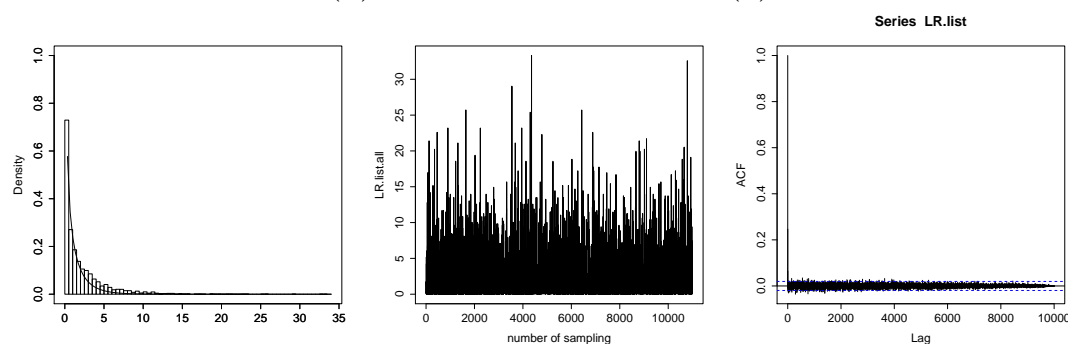

(c) a lattice basis with $P o(10)$
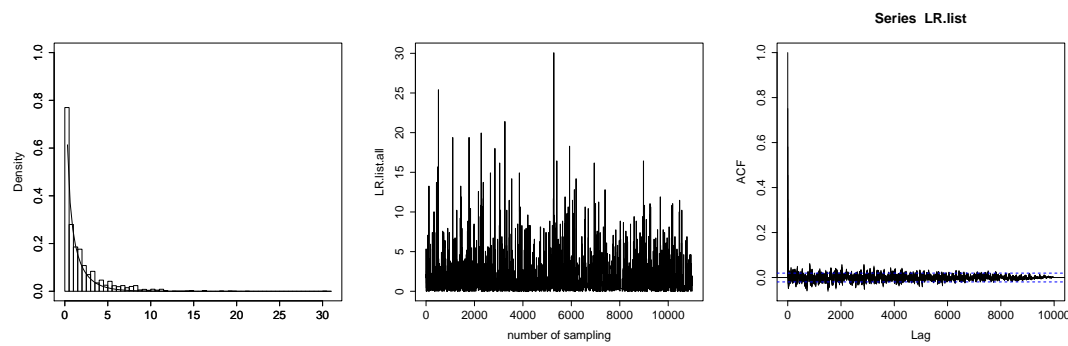

(d) a lattice basis with $P o(50)$

Fig. 3. Histograms, paths of LR statistic and correlograms of paths for discrete logistic regression model $(($ burn in,iteration $)=(1000,10000))$ 

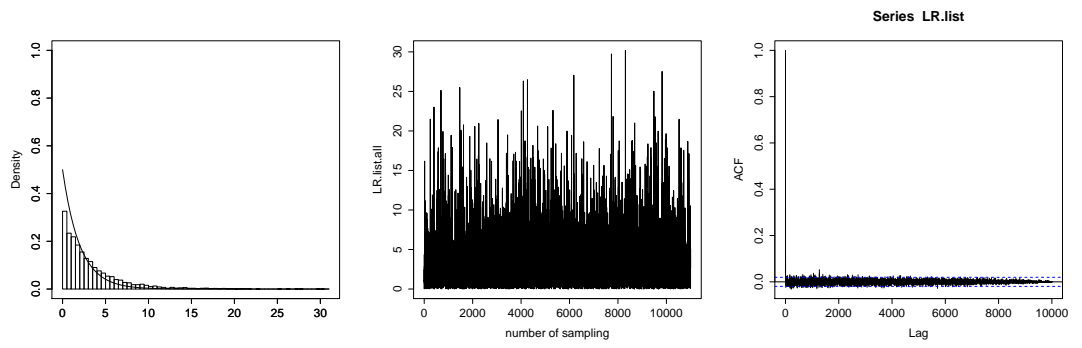

(a) a Markov basis
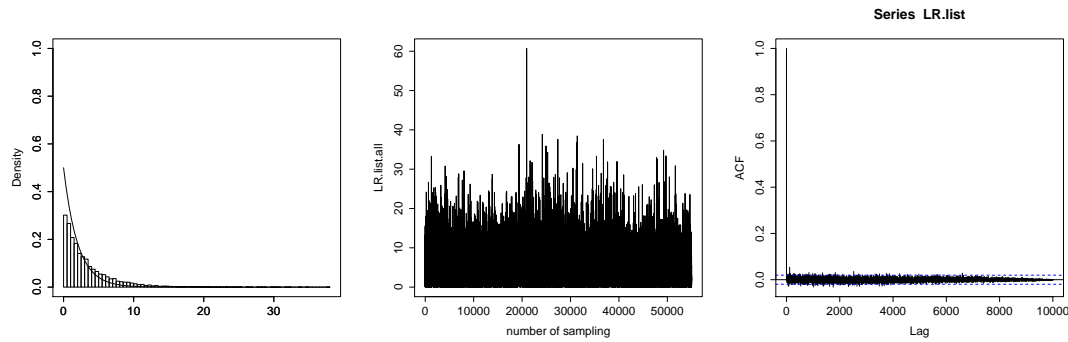

(b) a lattice basis with $P o(1)$
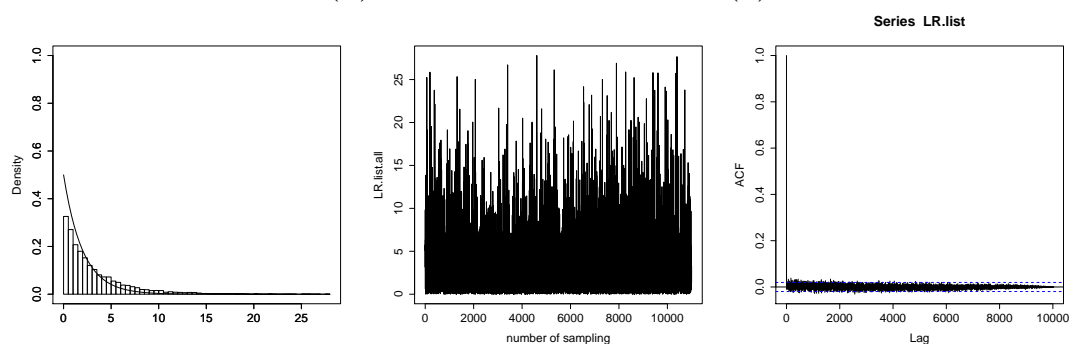

(c) a lattice basis with $P o(10)$
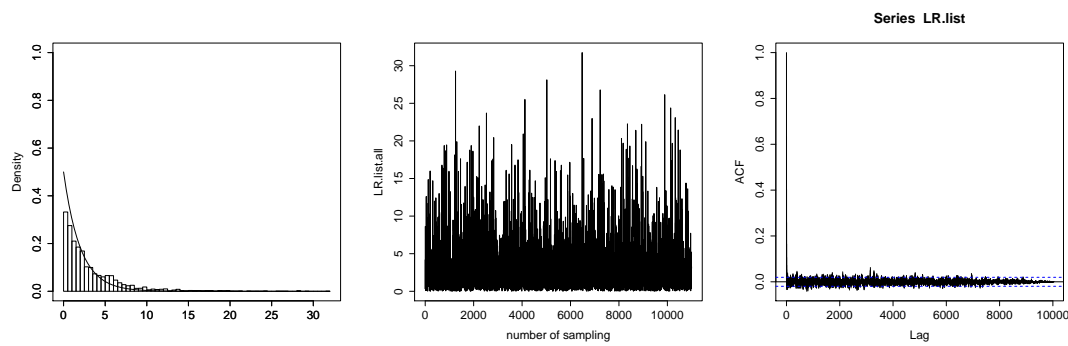

(d) a lattice basis with $P o(50)$

Fig. 4. Histograms, paths of LR statistic and correlograms of paths for trinomial discrete logit model $(($ burn in,iteration $)=(1000,10000))$ 

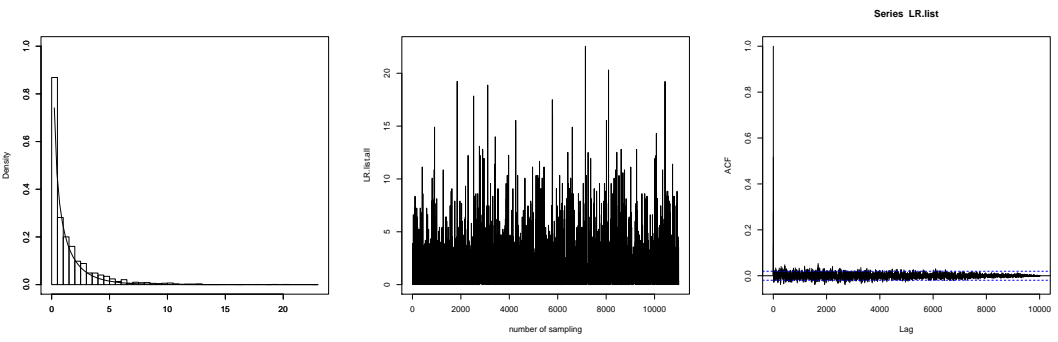

(a) binomial, a lattice basis with Geom(0.1)
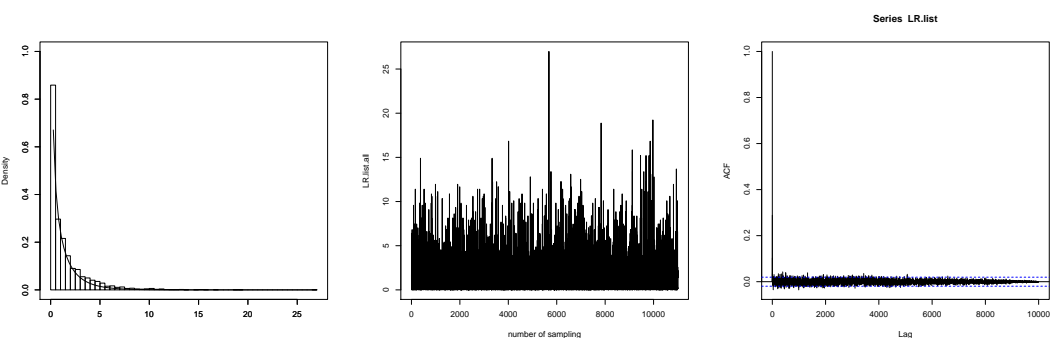

(b) binomial, a lattice basis with $\operatorname{Geom}(0.5)$
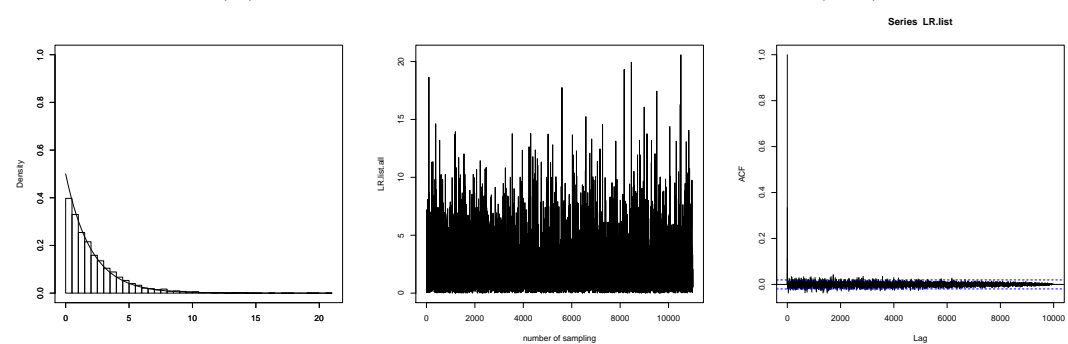

(c) trinomial, a lattice basis with Geom(0.1)
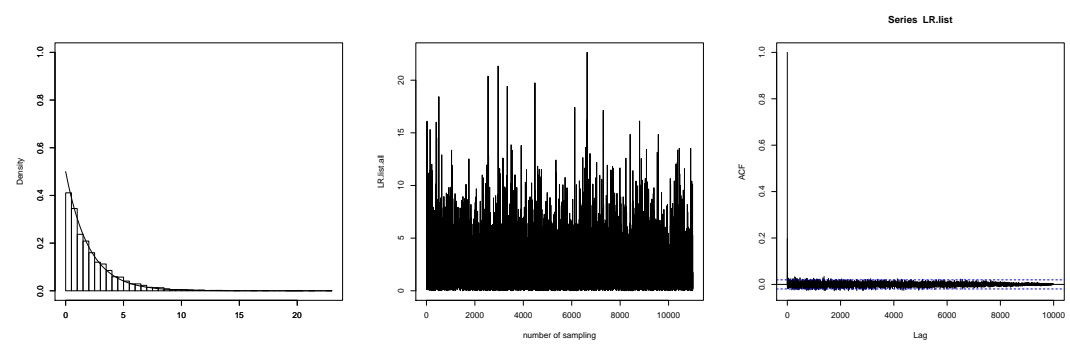

(d) trinomial, a lattice basis with Geom(0.1)

Fig. 5. Histograms, paths of LR statistic and correlograms of paths for discrete logistic regression model $(($ burn in,iteration $)=(1000,10000))$ 